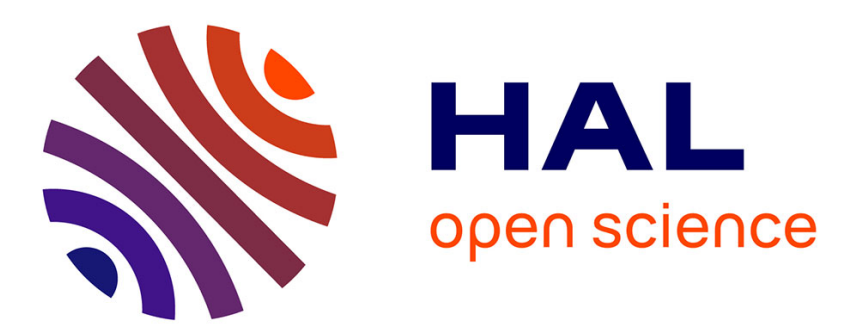

\title{
Neural basis of functional fixedness during creative idea generation: an EEG study
}

Anaëlle Camarda, Emilie Salvia, Julie Vidal, Benoit Weil, Nicolas Poirel, Olivier Houde, Gregoire Borst, Mathieu Cassotti

\section{- To cite this version:}

Anaëlle Camarda, Emilie Salvia, Julie Vidal, Benoit Weil, Nicolas Poirel, et al.. Neural basis of functional fixedness during creative idea generation: an EEG study. Neuropsychologia, In press. hal-01761372

\section{HAL Id: hal-01761372 \\ https://hal.science/hal-01761372}

Submitted on 9 Apr 2018

HAL is a multi-disciplinary open access archive for the deposit and dissemination of scientific research documents, whether they are published or not. The documents may come from teaching and research institutions in France or abroad, or from public or private research centers.
L'archive ouverte pluridisciplinaire HAL, est destinée au dépôt et à la diffusion de documents scientifiques de niveau recherche, publiés ou non, émanant des établissements d'enseignement et de recherche français ou étrangers, des laboratoires publics ou privés. 
Neural basis of functional fixedness during creative idea generation: an EEG study

\section{Anaëlle Camarda}

CNRS Unit 8240, Laboratory for the Psychology of Child Development and Education, Paris

Descartes University, Sorbonne Paris Cité University and Caen University, France

$$
\text { Émilie Salvia }
$$

CNRS Unit 8240, Laboratory for the Psychology of Child Development and Education, Paris Descartes University, Sorbonne Paris Cité University and Caen University, France

\section{Julie Vidal}

CNRS Unit 8240, Laboratory for the Psychology of Child Development and Education, Paris Descartes University, Sorbonne Paris Cité University and Caen University, France

\section{Benoit Weil}

Center for Management Science, Chair TMCI, Mines ParisTech, France

Nicolas Poirel

Institut Universitaire de France

\& CNRS Unit 8240, Laboratory for the Psychology of Child Development and Education,

Paris Descartes University, Sorbonne Paris Cité University and Caen University, France

\section{Olivier Houdé \\ Institut Universitaire de France}

\& CNRS Unit 8240, Laboratory for the Psychology of Child Development and Education, Paris Descartes University, Sorbonne Paris Cité University and Caen University, France

Grégoire Borst

Institut Universitaire de France

\& CNRS Unit 8240, Laboratory for the Psychology of Child Development and Education,

Paris Descartes University, Sorbonne Paris Cité University and Caen University, France

Mathieu Cassotti*

Institut Universitaire de France

\& CNRS Unit 8240, Laboratory for the Psychology of Child Development and Education,

Paris Descartes University, Sorbonne Paris Cité University and Caen University, France

Acknowledgments: This research was financed by a grant from the French National Research Agency (ANR-13-SOIN-0004-02 IDéfixE).

* Corresponding author:

Université Paris Descartes, Sorbonne, Laboratoire A. Binet

46 rue Saint Jacques

75005 Paris, France

Email: mathieu.cassotti@parisdescartes.fr

Tel.: +33140462995 


\begin{abstract}
Decades of problem solving and creativity research have converged to show that the ability to generate new and useful ideas can be blocked or impeded by intuitive biases leading to mental fixations. The present study aimed at investigating the neural bases of the processes involved in overcoming fixation effects during creative idea generation. Using the AU task adapted for EEG recording, we examined whether participant's ability to provide original ideas was related to alpha power changes in both the frontal and temporo-parietal regions. Critically, for half of the presented objects, the classical use of the object was primed orally, and a picture of the classical use was presented visually to increase functional fixedness (Fixation Priming condition). For the other half, only the name of the object and a picture of the object was provided to the participants (control condition). As expected, priming the classical use of an object before the generation of creative alternative uses of the object impeded participants' performances in terms of remoteness. In the control condition, while the frontal alpha synchronization was maintained across all successive time windows in participants with high remoteness scores, the frontal alpha synchronization decreased in participants with low remoteness scores. In the Fixation Priming condition, in which functional fixedness was maximal, both participants with high and low remoteness scores maintained frontal alpha synchronization throughout the period preceding their answer. Whereas participants with high remoteness scores maintained alpha synchronization in the temporo-parietal regions throughout the creative idea generation period, participants with low remoteness scores displayed alpha desynchronization in the same regions during this period. We speculate that individuals with high remoteness scores might generate more creative ideas than individuals with low remoteness scores because they rely more on internal semantic association and selection processes.
\end{abstract}


Neural basis of functional fixedness during creative idea generation

Decades of problem solving and creativity research have converged to show that the ability to generate new and useful ideas (Sternberg \& Lubart, 2001) can be blocked or impeded by intuitive biases leading to mental fixation (Adamson, 1952; Agogué et al., 2014; Agogué, Poirel, Pineau, Houdé, and Cassotti, 2014; Duncker, 1945). Although fixation effects might often be useful during problem solving where creative thinking is not required, they will sometimes cue the classical paths of uncreative solutions that constrain the exploration of alternative original ideas (Adamson, 1952; Agogué, Kazakci, et al., 2014; Agogué, Poirel, et al., 2014; Cassotti et al., 2016; Duncker, 1945; Purcell \& Gero, 1996; Smith; Ward, and Schumacher, 1993; Ward, 1994). Although several previous experimental studies and theories have argued that creative idea generation exclusively involves automatic processes such as incubation, defocused attention or mind-wandering (Martindale, 1999; Radel, Davranche, Fournier, and Dietrich, 2015), recent behavioral and neuroimaging findings have shown that the difficulty in generating creative ideas to a problem results from a failure to inhibit intuitive responses based on the activation of the most common and accessible knowledge within a specific task (Benedek, 2012; Cassotti, Agogué, Camarda, Houdé, and Borst, 2016; Kleibeuker, De Dreu, and Crone, 2016). Put bluntly, these studies suggest that to think outside of the box, one must first inhibit what is inside the box (Camarda et al., in press; Cassotti et al., 2016; Storm and Angello, 2010). Although considerable effort has been recently devoted to identifying the cognitive processes involved in creative thinking, to date, few studies have investigated the neurocognitive processes involved in overcoming fixation effects in creative ideation. The present study aimed at examining the neural bases of mental fixation and the processes that allow overcoming this bias to generate original solutions in a creative task using EEG recordings. 
The seminal work of Duncker (1945) was the first to reveal that the ability to explore unusual solutions during problem solving can be constrained by the activation of the typical function of an object. This is typically the case in the candle problem (Duncker, 1945; Adamson, 1952; German \& Defeyter, 2003; German \& Barrett, 2005) in which participants are asked to fasten a candle on a door in such a way that it will burn without dripping wax onto the table below. To do so, they must use objects available to them, including a candle, a box of thumbtacks and matches. To solve the problem, participants must use the box in an unfamiliar way - i.e., as a platform for the candle (by attaching the box to the door using the thumbtacks). Duncker (1945) observed that participants were better at solving the task when the box was presented empty (control condition) than when it was full of thumbtacks, which primed the common use of the box. Thus, when participants are primed with the common function of the box, it impedes their ability to use the box in an atypical manner. These results suggest that priming the classical use of an object leads to functional fixedness, which in turn constrains the ability to find an original solution to the candle problem (Duncker, 1945; Adamson, 1952; German \& Defeyter, 2003; German \& Barrett, 2005). A similar fixation effect on the most accessible and common knowledge has been reported when individuals have to generate ideas rather than solve a problem (Ward, 1994; Agogué, Poirel, et al., 2014; Agogué et al., 2015; Purcell \& Gero, 2006; Jansson \& Smith, 1991). For example, when they are asked to draw an animal that lives on another planet very different from Earth, participants tend to focus on the seminal characteristics of animals living on Earth, such as having a bilateral symmetry, sensory organs (e.g., eyes or ears), and body parts (e.g., legs or wings, Karminoff -Smith, 1990; Smith, Ward \& Schumacher, 1993; Ward, 1994; Kohn \& Smith, 2011). Taken together, these findings suggest that participants follow the "path of least resistance" to minimize the cognitive cost associated with the task by activating knowledge bases close to the generative concept. Thus, the mental fixation observed during creativity 
tasks might not reflect an inability to generate original solutions per se but rather a failure to inhibit intuitive solutions based on the spontaneous activation of common knowledge (Agogué, Poirel, et al., 2014; Agogué et al., 2015, Cassotti et al., 2016, Camarda et al., in press). In line with this hypothesis, a growing number of studies have demonstrated that creative idea generation is related in part to inhibitory control (i.e., the ability to resist to impulses, prevalent thoughts, knowledge and behaviors, see Diamond, 2013), as suggested by the positive correlations reported between inhibition measures and divergent thinking performance (Beaty, Silvia, Nusbaum, Jauk, \& Benedek, 2014; Benedek, et al., 2012; Vartanian, 2009). For example, Benedek et al. (2014) examined the relations between creative performance (i.e., divergent thinking ability), fluid intelligence and three executive functions (i.e., inhibitory control, updating and shifting). The results revealed that updating (but not inhibitory control or shifting) predicted fluid intelligence. Critically, updating and inhibitory control (but not shifting) predicted creative idea generation, suggesting that specific executive functions are related to creative ideation. A recent study that used a dual-task approach to reduce participants' inhibitory control resources while performing a creative task provided additional evidence for the role of inhibitory control in creative idea generation (Camarda et al., in press) - i.e., inhibitory control load decreased creative capabilities in terms of fluidity, flexibility and originality in this study. Taken together, these findings converge to show that inhibitory control is a core process that allows one to overcome fixation effects and generate original solutions in a creative task (Cassotti et al., 2016). Consistent with this view, a recent neurocognitive model of creativity assumes that creative ideation involves dynamic interactions between the default and the executive control networks. More specifically, this model suggests that "the default network influences the generation of candidate ideas, but that the control network can constrain and direct this process to meet task-specific goals via topdown monitoring and executive control” (Beaty, Benedek, Silvia, and Schacter, 2016). 
The findings of electrophysiological studies also support the claim that high-level cognitive processes are required during ideation, as revealed by the relation observed between the variations in alpha power during EEG (electroencephalography) and ideation processes (Benedek, Bergner, Könen, Fink, Grabner, Benedek, \& Neubauer, 2011; Benedek, et al., 2012). Indeed, previous EEG studies revealed that an increase in alpha power (alpha synchronization) reflects the activation of top-down processes, while a decrease in alpha power (alpha desynchronization) reflects the activation of bottom-up processes (Pfurtscheller, 1999; Pfurtscheller, Stancak \& Neuper, 1996; for a review, see also Klimesch et al., 2007). For example, a high level of alpha power is observed when individuals have to keep information in memory (Sauseng, Klimesch, Schabus, and Doppelmayr, 2005) or when they have to focus their attention on internal processes rather than on external stimuli (Cooper, Croft, Dominey, Burgess \& Cruzelier, 2003; Sauseng et al., 2005; Von Stein \& Sarnthein, 2000; Benedek et al., 2011). To determine the role of top-down or bottom-up processes in creativity, a series of studies (Benedek et al., 2011; Benedek et al., 2014) examined the variations in alpha power during the execution of creative tasks, including an emblematic divergent thinking task called the «Alternative Uses Task » (AU task, Guilford, 1966). These studies used an adaptation of the AU task in which participants were asked to give alternative use of a common object (e.g., a brick) while the name of this object was displayed (low internal condition) or not (high internal condition) on a computer screen. The authors analyzed a period of $1 \mathrm{~s}$ preceding the participants' answers and observed an alpha desynchronization in the posterior regions of the brain when participants used bottom-up information (low internal condition) and an alpha synchronization in the bilateral prefrontal regions and right temporo-parietal regions when the task required them to maintain the name of the object in working memory (high internal condition). These results suggest the existence of a functional dynamic between prefrontal regions (typically involved in top-down processes 
such as inhibitory control) and right temporo-parietal regions (which are associated with highlevel semantic processes) (Fink et al., 2007; Benedek et al., 2011; 2014; Martindale \& Hasenful, 1978). In addition, the bilateral prefrontal and the right temporo-parietal alpha synchronization was related to the originality of the ideas generated (Fink, Grabner, Benedek, \& Neubauer, 2006; Fink et al., 2009). To better characterized how EEG recordings might reflect the different processes involved during a creative task, Schwabs, Benedek, Papousek, Weiss, and Fink (2014) asked their participants to generate an alternative solution every 10 seconds. By dividing this period into 3 different and consecutive time windows of analysis, they observed a decrease of alpha synchronization in the left hemisphere, while a U-shaped pattern was observed in the right hemisphere in the frontal and parietal regions. According to the authors, this alpha synchronization pattern revealed that the generation of creative ideas relies on 1) memory recall of common ideas, 2) internally directed attention-facilitating imaginative processes and 3) working memory involved in the search for different information within the semantic network. Moreover, this pattern was more pronounced in higher original ideas compare to less original ideas, indicating that ideas of high quality might involve the same neural mechanisms as lower quality ideas but in a more efficient manner. Although this study highlights the relevance of studying alpha power in different time windows preceding the generation of creative ideas, the extent of the time windows specified (10 seconds) might not allow for the identification of the cognitive processes supporting creative idea generation per se but more domain general processes such as the retention of information in memory or the choice between the different responses generated.

In the present study, we investigated the neural bases of the specific processes involved in overcoming a fixation during the generation of creative ideas by using smaller time windows of interest in EEG (200 ms). Participants performed under two conditions. Participants were asked to generate alternative solutions regarding the use of an object (as in a 
classical AU task, Benedek et al., 2011; Benedek et al., 2012; Guilford, 1967) while the name of the object was presented before the generation of the idea (Control condition) or while the name and the usual use of the object were presented before the generation of the idea (Fixation Priming condition). In line with findings from previous studies (Adamson, 1952; Duncker, 1945; German \& Defeyter, 2003; German \& Barrett, 2005), priming the classical use of an object should increase the fixation effect and the cognitive cost related to overcoming this fixation effect (Cassotti et al., 2016). Thus, we expected participants to generate more creative ideas in the Control than in the Fixation Priming condition. In addition, a fine-grained analysis of the alpha power temporal dynamics should allow us to study the neural basis of the specific processes involved in overcoming the fixation effect. We hypothesized that if executive control and semantic processes are the two key mechanisms allowing one to generate ideas outside of the fixation effect, as predicted by recent models of creativity (Beaty et al., 2016; Cassotti et al., 2016), then we should observe a greater synchronization of the alpha power in frontal (typically involved in top-down processes such as inhibitory control) and temporo-parietal (which are associated with high-level semantics processes) regions of the brain during the Fixation Priming condition than in the Control condition. Moreover, based on previous EEG studies (Schwab et al., 2014), we expected to observe this pattern more strongly for participants with high creativity scores than for participants with low creativity scores. To further identify the nature of the processes allowing one to overcome the fixation effect, we asked participants to perform the Hayling Sentence Completion Test (Burgess \& Shallice, 1996), a test of semantic inhibition (Burgess \& Shallice, 1996; Volle et al., 2012). We reasoned that if semantic inhibition is required to overcome the fixation effect during creative idea generation, then the scores in the Hayling Sentence Completion Test should be correlated with the creativity of the ideas generated. 
Method

\section{Participants}

Twenty-four healthy adults participated in this experiment. Three participants were excluded from data analyses due to excessive artifacts in the EEG signal. The final sample included 21 participants ( 11 females and 10 males, $M=20.33, S D=2.18$ ). All participants were right-handed. They had normal or corrected-to-normal vision. They indicated no history of psychiatric or neurological disorders or treatment that could have interfered with both the behavioral and neurophysiological measures. All participants provided written informed consent and were tested in accordance with national and international norms governing the use of human research participants.

Inhibitory control task

During the Hayling Sentence Completion Test (Burgess \& Shallice, 1996), participants had to complete 15 short sentences with the last word missing but easily found through the first part of the sentence. In one condition, they had to provide the correct answer (congruent condition) while in a second condition they had to provide a word completely unrelated to the sentence (incongruent condition). For example, for the sentence: "He sent the letter without a...", the participant would answer "stamp" in the congruent condition, while he could answer "cream" in the incongruent condition. Thus, in contrast with the congruent condition, in the incongruent condition, participants had to inhibit a strong automatic response (e.g., stamp) before generating an unrelated word (e.g., cream).

Reaction times (RTs) were recorded in seconds using a standard electronic stopwatch and corresponded to the time between the end of the sentence given by the experimenter and the answer of the participant. We converted answer given by participants into scaled scores based on the Pérez-Pérez et al., (2016) scoring system as follows: for the congruent 
conditions, answers were scored as correct ( 0 points) or incorrect (1 point); and for the incongruent conditions, answers were scored as answer congruent with the sentence (3 points), answer semantically related or opposite (1 point) or answer incongruent with the sentence (0 point) (Pérez-Pérez et al., 2016). Consequently, lower RTs and scores reflect better semantic inhibition abilities. Then, an interference score (incongruent condition score congruent condition score) and interference RT (incongruent condition RT - congruent condition RT) were calculated, allowing the measurement of the efficiency of inhibitory control.

\section{Creative task}

We used the AU task (Guilford, 1967), which has already been successfully used in neuroimaging studies (Fink, Grabner, Benedek, and Neubauer, 2006; Grabner, Fink, and Neubauer, 2007) and allows the measurement of divergent thinking. In this task, everyday objects (e.g., "bike" or "umbrella") were presented visually on a screen (black and white drawings displayed on the screen) accompanied by the soundtrack of a female voice providing the name of the object (Control condition) or the name and the classical use of the object (Fixation Priming condition). Participants were instructed to generate a maximum original, unconventional ideas on how to use these objects in 2 minutes (i.e., idea generation phase). Fourteen objects were selected and presented to each participant.

Participants completed the AU task under two experimental conditions: a Control condition (i.e., presentation of the name of the object before the generation phase; $\mathrm{C}_{\mathrm{C}}$ ); and a Fixation Priming condition (i.e., presentation of both the name and the classic use of the object before the generation phase; $\mathrm{C}_{\mathrm{FP}}$ ). In each experimental condition, participants were exposed to seven different objects. Two versions of the experiment were designed to counterbalance (i) the presentation of objects (i.e., 7 out of 14) in each condition and (ii) the 
order of presentation of these objects in each condition. For instance, the object "Hat" was presented in the control condition for half of the participants (i.e., the object was presented as "a hat"), and it was presented in the Fixation Priming condition for the other half of the participants (i.e., the object was presented as "a hat to protect a head").

As shown in Figure 1, the AU task started with the presentation of a fixation cross for a duration of 5 s (i.e., a baseline period). Then, the audio-visual presentation of the object for which participants had to generate original uses appeared on the screen until the participant pressed a button to notify that he / she got an idea. The participant was then prompted to provide his / her idea orally and then press a button. After the button press, the object (and the classical use in the Fixation Priming condition) reappeared on the screen. The object (and its classic use in the Fixation Priming condition) were presented in different formats: (i) the name of the object (as well as its classic use in the Fixation Priming condition) was displayed on the screen, (ii) a black and white drawing depicting the object (and its classic use in the Fixation Priming condition), and (iii) a female voice describing the object (and its classic use in the Fixation Priming condition). The stimuli were presented using E-Prime (Psychology Software Tools, Pittsburgh, PA) on a computer with a screen resolution of $1024 * 768$ pixels $(340 * 270 \mathrm{~mm})$.

The performance of the participants on the AU task was quantified by means of a fluency score (i.e., the capacity to generate numerous solutions) and an originality score (i.e., the quality of the produced ideas; Fink et al., 2010). We further added a new measure of the creative outputs, which is referred to as « remoteness » and reveals the distance from the given responses relative to the classic use of the object (i.e., the ability to generate ideas based on remote associations). 
To measure fluency, we counted the number of solutions that were provided by the participants. When a participant proposed a solution that was a combination of different proposals, we counted each proposal as one solution.

To assess originality and remoteness, we applied an external rating procedure that was similar to the consensual assessment technique proposed by Amabile (1982). More specifically, two independent raters were instructed to evaluate each idea on a five-point rating scale ranging from 1 (not original at all) to 5 (highly original) for the originality scores and from 1 (close to the classic use of the object) to 5 (far from the classic use of the object) for the remoteness scores. Originality and remoteness scores were assessed for each participant by averaging the ratings for all solutions. The raters were generally in agreement on their ratings on these originality and remoteness scores, as suggested by the satisfactory intraclass correlation coefficients (ICC) computed for each score (for originality: $\mathrm{ICC}=.77$; for remoteness: ICC $=.78$ ). A correlation analysis between our different measures of creativity revealed that remoteness and originality scores are highly correlated $(r(19)=.69, \mathrm{p}$ $<.001)$. Given that the remoteness and originality scores were highly correlated, we restricted the data analysis to the remoteness scores

\section{(PLEASE INSERT FIGURE 1 ABOUT HERE)}

\section{Procedure}

Participants first underwent a training phase. Three objects (different than those presented in the experiment) were presented, as in the experimental phase, to familiarize the participants with the procedure and, in particular, to getting used to pressing the button as soon as an idea came to mind while minimizing their movements during the task. 
The experimental session took place in a Faraday cage that did not enable the experimenter to stay in the same room as the participant. However, a window allowed the experimenter to maintain visual contact with the participants and the participants to communicate with the experimenter if necessary. Participants were asked to complete the AU task by providing their responses orally. Note that the influence of social factors on creative idea generation was limited due to the Faraday cage (Amabile et al., 2012). Each of the participants' responses were recorded. The room's brightness was reduced to allow the participants to focus on the task presented on the screen and thus reduced their eye movements towards the environment. The experimental session was split in two blocks with a pause in-between. Each block included the presentation of seven objects. In total, the duration of the experiment did not exceed one hour. Then, outside the Faraday cage, an experimenter asked participants to complete the Hayling Test, which required approximately ten minutes to complete.

EEG data recording and analysis

Electroencephalograms (EEG) were recorded from a 256-channel HydroCel Geodesic Sensor Net (Electrical Geodesics Inc., Eugene, Oregon, USA) containing electrodes imbedded in small sponges soaked in a potassium chloride saline solution. Continuous EEGs were acquired through a DC amplifier (Net Amps 300 1.0.1, EGI) and digitized at a sampling rate of $1000 \mathrm{~Hz}$. A common reference at the vertex was used during acquisition, and electrode impedances were kept below $100 \mathrm{k} \Omega$. Eye-blinks and eye-movements were monitored via pairs of channels included in the net and covering the face area. The processing steps described below were performed using EEGLab (Delorme \& Makeig, 2004).

Activity from all electrodes was re-referenced to the average. The signal was then down-sampled to $500 \mathrm{~Hz}$. The raw EEG data were passed through a high-pass filter $(0.1 \mathrm{~Hz})$, 
a low-pass filter $(100 \mathrm{~Hz})$ and a Notch filter $(50 \mathrm{~Hz})$ to avoid power line contaminations. Muscular artifacts and ocular artifacts were removed from continuous EEG data using Artifact Subspace Reconstruction (ASR, implemented in an EEGLab plugin, i.e., clean_rawdata - Mullen et al., 2015). The continuous EEG data were then segmented from $1250 \mathrm{~ms}$ to $-250 \mathrm{~ms}$ relative to the onset of each participant's response (i.e., idea generation). For each participant, segments were binned into two categories: control (i.e., $\mathrm{C}_{\mathrm{C}}$ ) or fixation priming (i.e., $\left.\mathrm{C}_{\mathrm{FP}}\right)$. After artifact rejection, we preserved a mean of $17.8(\mathrm{SD}=5.98)$ and $15.20(\mathrm{SD}=4.43)$ segments of idea generation per participant for the $\mathrm{C}_{\mathrm{C}}$ and $\mathrm{C}_{\mathrm{FP}}$ conditions, respectively. We also segmented the continuous EEG data from $500 \mathrm{~ms}$ to $4500 \mathrm{~ms}$ relative to the onset of the presentation of the fixation cross preceding the first occurrence of each object, which was the baseline period and occurred before each new object. A unique baseline (reference) period per object was recorded.

For time-frequency spectral analyses, Morlet wavelet transformations, as implemented in EEGLab (Delorme \& Makeig, 2004), were applied. Signal power (i.e., the amplitude squared) was computed for frequencies between 8.5 and $12.5 \mathrm{~Hz}$ using a constant wavelet parameter of 3 cycles and a $0.5 \mathrm{~Hz}$ frequency step. We focused on the alpha power, as this frequency band is particularly sensitive to creative processes (Benedek et al., 2011). Changes in cortical activation were defined as the task-related power (TRP) changes between baseline (i.e., fixation cross) and activation (i.e., idea generation) periods for each electrode and condition (Pfurtscheller \& Lopes da Silva, 1999). As the variance of spectral power measures can be stabilized by logarithmic (log) transformations (Halliday et al., 1995), task-related power at an electrode i was computed by subtracting the log-transformed power during the baseline interval ( Pow $_{\mathrm{i}}$, baseline) from the log-transformed power during the idea generation interval, which was the activation period $\left(\mathrm{Pow}_{\mathrm{i}}\right.$, activation $)$, according to the formula: TRP $(\mathrm{i})=$ $\log \left(\right.$ Pow $\left._{\mathrm{i}, \text { activation }}\right)-\log \left(\right.$ Pow $\left._{\mathrm{i}, \text { baseline }}\right)$ (see Fink et al., 2006; Benedek et al., 2011, 2014). 
Negative values reflect decreases in power during the idea generation interval compared to the baseline interval (referred to as desynchronization) while positive values reflect increases in power during the idea generation interval (referred to as synchronization; Pfurtscheller \& Lopes da Silva, 1999).

As this study seeks to investigate the temporal dynamics of idea generation at both frontal and temporo-parietal sites, the data were processed as follows: (i) the idea generation interval (i.e., $1000 \mathrm{~ms}$, from $-1250 \mathrm{~ms}$ to $-250 \mathrm{~ms}$ preceding the participants' response) was split into 9 time windows of $200 \mathrm{~ms}$ each, overlapping by $100 \mathrm{~ms}$; and (ii) the TRP data were aggregated from 4 different subsets of electrode positions as shown in Figure 2. Given that previous EEG studies on creative idea generation have supported the hypothesis of the existence of a functional dynamic between prefrontal regions (typically involved in top-down processes such as inhibitory control) and temporo-parietal regions (which are associated with high-level semantic processes) (Fink et al., 2007; Benedek et al., 2011; 2014; Martindale \& Hasenful, 1978), we have created 4 distinct ROIs to examine how experimental conditions influence the TRP during idea generation. Based on ROIs characterized in previous EEG studies (Benedek et al., 2011; Jauk, Benedek and Neubauer, 2012), we have determined two frontal regions of interest with 20 electrodes each (see Figure 2: 1-left frontal ROI, 2-right frontal) and two temporo-parietal regions of interest with 23 electrodes each (see Figure 2: 3left temporo-parietal ROI, 4-right temporo-parietal ROI).

\section{(PLEASE INSERT FIGURE 2 ABOUT HERE)}

\section{Statistical analyses}

First, to determine whether priming fixation using the presentation of both the name and the classic use of the object before the generation phase reduces fluency and remoteness, 
we conducted t-tests between the mean scores of fluency and remoteness computed on all the responses provided by the participants in the Fixation Priming condition $\left(\mathrm{C}_{\mathrm{FP}}\right)$ and the Control condition $\left(\mathrm{C}_{\mathrm{C}}\right)$. Significance thresholds were set to $\mathrm{p}<.05$. In addition, to examine the neurocognitive processes involved in overcoming functional fixedness during creative idea generation, we conducted analyses of variance on the remoteness scores, considering both the response type $\left(1^{\text {st }}\right.$ response vs. last response provided by the participants $)$ and condition $\left(\mathrm{C}_{\mathrm{C}}\right.$ vs. $\left.\mathrm{C}_{\mathrm{FP}}\right)$ as within-subjects factors. We compared remoteness scores on the first response to the ones on the last response because (a) later responses tend to be more creative than earlier ones in divergent thinking tasks (Beaty et al., 2014; Christensen, Guilford, \& Wilson, 1957), and (b) this serial order effect might rely on an increasing demand on executive control during idea generation (Beaty et al., 2014, Camarda et al., in press; Zabelina \& Robinson, 2010, see for review Beaty et al., 2016; Cassotti et al., 2016). For example, Hao, Wu, Runco and Pina (2015) reported that the demand on executive control is higher for the last responses compared to the first ones in a creative task.

Given that previous EEG studies on creative ideation have revealed that the generation of highly original ideas (assessed using a median split based on the originality of the responses given by the participants) are associated with stronger task-related alpha synchronization in the frontal and parietal brain regions (Fink \& Benedek, 2014; Fink et al., 2006), we used a median split to divide the sample in two groups. Participants with high remoteness scores $(n=10 ; M=3.0, S D=0.27)$ were compared to participants with low remoteness ones $(\mathrm{n}=11 ; \mathrm{M}=2.08, \mathrm{SD}=0.38 ; \mathrm{t}(1,19)=5.99, \mathrm{p}<.001)$. It should be noted that the task-related power (TRP) was obtained by using all the responses given by the participants. Then, we conducted an ANOVA on TRP considering Condition $\left(\mathrm{C}_{\mathrm{C}}\right.$ vs. $\left.\mathrm{C}_{\mathrm{FP}}\right)$, ROI (frontal vs. temporo-parietal), Laterality (right vs. left) and Time Window (see Figure 4) as within-subjects factors and Remoteness (participants with high remoteness vs. participants 
with low remoteness scores) as a between-subjects factor. For all ANOVAs, Mauchly test of sphericity was applied. When sphericity assumption was violated, we used a GreenhouseGeisser correction. We used partial eta squared $\left(\eta_{\mathrm{p}} 2\right)$ to assess the effect size.

\section{Results}

Behavioral results

A t-test did not reveal a significant difference between the average number of responses given in the control condition $\left(\mathrm{MC}_{\mathrm{C}}=5.57, \mathrm{SD}=2.32\right)$ and in the fixation priming condition $\left(\mathrm{MC}_{\mathrm{FP}}=5.29, \mathrm{SD}=2.31 ; \mathrm{t}(20)=1.22, \mathrm{p}=.23, \mathrm{~d}=.12\right)$. Similar results were obtained for the remoteness scores $(\mathrm{t}(20)<1)$.

We conducted a repeated-measures ANOVA on the remoteness scores (i.e., distance to fixation) considering both the response type ( $1^{\text {st }}$ response vs. last response provided by the participants $)$ and condition $\left(\mathrm{C}_{\mathrm{C}}\right.$ vs. $\left.\mathrm{C}_{\mathrm{FP}}\right)$ as within-subjects factors. We found a significant main effect of the response type, with the distance to fixation being higher for the last response than for the first one $\left(\mathrm{F}(1,20)=6.23, \mathrm{p}=.02, \eta_{\mathrm{p}}{ }^{2}=.23\right)$, while no significant main effect of the condition was revealed $\left(\mathrm{F}(1,20)=0.99, \mathrm{p}=.33, \eta_{\mathrm{p}}{ }^{2}=.05\right)$. We also found a significant interaction between the response type and the condition $(F(1,20)=4.52, p=.045$, $\eta_{\mathrm{p}}{ }^{2}=.18$, see Figure 3 ). While in the $\mathrm{C}_{\mathrm{C}}$, remoteness did not differ between the first response and the last one $\left(\mathrm{M}_{\text {first response }}=2.54, \mathrm{SD}=0.85 ; \mathrm{M}_{\text {last response }}=2.52, \mathrm{SD}=0.83 ; \mathrm{t}(20)<1\right)$, in the $\mathrm{C}_{\mathrm{FP}}$, the first response was closer to the fixation (i.e., classic use of the object) than the last one $\left(\mathrm{M}_{\text {first response }}=2.17, \mathrm{SD}=0.63 ; \mathrm{M}_{\text {last response }}=2.64, \mathrm{SD}=0.85 ; \mathrm{t}(20)=3.77, \mathrm{p}=.001, \mathrm{~d}=\right.$ .62). Furthermore, participants had more difficulties getting rid of the classic use of the object in the $\mathrm{C}_{\mathrm{FP}}\left(\mathrm{M}_{\mathrm{FP}}=2.17, \mathrm{SD}=0.63\right)$ than in the $\mathrm{C}_{\mathrm{C}}\left(\mathrm{MC}_{\mathrm{C}}=2.54, \mathrm{SD}=0.85 ; \mathrm{t}(20)=2.80, \mathrm{p}=\right.$ $.01)$ for the first response but not for the last response $\left(\mathrm{MC}_{\mathrm{C}}=2.52, \mathrm{SD}=0.14 ; \mathrm{M}_{\mathrm{FP}}=2.64\right.$, $\mathrm{SD}=0.19 ; \mathrm{t}(20)=0.58, \mathrm{p}=.56)$. 
To investigate the extent to which creative performances were related to inhibitory control, we conducted a correlation analysis to test the relation between AU remoteness scores and measures obtained from the Hayling Sentence Completion Test (i.e., RTs and scores). While the correlation between AU remoteness scores and Hayling scores was marginally significant $(\mathrm{r}=.44, \mathrm{p}=.056)$, we found a significant correlation between the remoteness scores in the AU task and RTs $(r=.61, p<.01$; see Figure 3$)$, suggesting that the ability to generate creative ideas might be related in part to the inhibition of semantically irrelevant information.

\section{(PLEASE INSERT FIGURE 3 ABOUT HERE)}

\section{Electrophysiological results}

We conducted an ANOVA on TRP considering Condition $\left(\mathrm{C}_{\mathrm{C}}\right.$ vs. $\left.\mathrm{C}_{\mathrm{FP}}\right)$, ROI (frontal vs. temporo-parietal), Laterality (right vs. left) and Time Window as within-subjects factors and remoteness (participants with high remoteness vs. participants with low remoteness scores) as a between-subjects factor ${ }^{1}$. We found significant main effects of the following: (i) ROI, with the alpha synchronization being larger in the frontal region than in the temporoparietal one $\left(\mathrm{F}(1,19)=7.88, \mathrm{p}=.01, \eta_{\mathrm{p}}^{2}=0.29\right)$; (ii) laterality, with the alpha

\footnotetext{
${ }^{1}$ Given that some previous EEG studies of creative ideation reported that the upper alpha band is more sensitive to high task demands than the lower alpha band (Fink \& Benedek, 2014; Klimesch, Sauseng, \& Hanslmayr, 2007), we included this factor (lower vs. upper alpha band) in the general ANOVA on the TRP. However, the Group $x$ ROI $x$ Condition $x$ Time Window $x$ Alpha band failed to reach significance $\left(F(8,152)=0.29, p=.97, \eta_{p}{ }^{2}\right.$ $=0.01)$. Critically, results confirmed that findings of both alpha bands are similar with a significant Group $x$ ROI $x$ Condition $x$ Time Window interaction for the upper alpha band $\left(F(8,152)=2.10, p=.04, \eta_{p}{ }^{2}=0.10\right)$ and the lower alpha band band $(F(8,152)=2.14, p=$ $\left..04, \eta_{p}^{2}=0.10\right)$.
} 
synchronization being larger in the right hemisphere than in the left one $(F(1,19)=33,67, p<$ $.001, \eta_{\mathrm{p}}^{2}=0.64$ ); (iii) time window, with the alpha TRP showing a progressive desynchronization over the analysis window (i.e., $1000 \mathrm{~ms} ; \mathrm{F}(8,152)=4.34, \mathrm{p}<.001, \eta_{\mathrm{p}}{ }^{2}=$ 0.19); and (iv) remoteness, with the participants with high remoteness scores showing a larger alpha synchronization than participants with low remoteness scores $(F(1,19)=6.21, p=.02$, $\left.\eta_{\mathrm{p}}^{2}=0.24\right)$. However, no significant main effect of the condition was observed $(\mathrm{F}(1,19)=$ 0.56, $\mathrm{p}=.46, \eta_{\mathrm{p}}{ }^{2}=0.02$ ). We also found a significant Condition $\mathrm{x}$ ROI $\mathrm{x}$ Time Window $\mathrm{x}$ Remoteness interaction $\left(F(8,152)=2.15, \mathrm{p}=.03, \eta_{\mathrm{p}}{ }^{2}=0.10 \text {; see Figure } 4\right)^{2}$.

\section{(PLEASE INSERT FIGURE 4 ABOUT HERE)}

To quantify this interaction, we conducted linear and quadratic trend analyses on the different time windows for each condition $\left(\mathrm{C}_{\mathrm{C}}\right.$ vs. $\left.\mathrm{C}_{\mathrm{FP}}\right)$ and $\mathrm{ROI}$ (frontal vs. temporo-parietal).

In the $\mathrm{C}_{\mathrm{C}}$ condition, participants with high remoteness scores showed a progressive decrease in frontal alpha TRP over the analysis window as revealed by a significant linear trend $(F(1,19)=10.11, p=.005)$, while no signal decrease following either a linear $(F(1,19)$ $<1, \mathrm{p}=.86)$ or a quadratic $(\mathrm{F}(1,19)=1.54, \mathrm{p}=.24)$ trend was observed for participants with high remoteness scores. Participants with high remoteness scores showed an alpha synchronization along different time windows. Similarly, participants with high remoteness scores did not show desynchronization over the different time windows in the temporoparietal region $\left(\mathrm{F}_{\text {Linear }}(1,19)<1 ; \mathrm{F}_{\text {quadratic }}(1,19)=2.10, \mathrm{p}=.16\right)$. However, participants

\footnotetext{
${ }^{2}$ Given the low sample size, we conducted a mixed-effect regression analysis on TRP using remoteness scores as a continuous variable and considering Condition (CC vs. CFP), ROI (frontal vs. temporo-parietal), Laterality (right vs. left) as within-subjects factors. Results confirmed a significant Condition $x$ ROI $x$ Remoteness interaction $(b=0.04 ; t(144.35)=$ 2.17, $p=.032$ ).
} 
with low remoteness scores showed a marginally linear desynchronization pattern in the temporo-parietal region $(\mathrm{F}(1,19)=3.24, \mathrm{p}=.08)$.

Results from the $\mathrm{C}_{\mathrm{FP}}$ condition did not reveal signal variations following linear trends in the frontal region $\left(\mathrm{F}_{\text {participants with low remoteness }}(1,19)=2.62, \mathrm{p}=.11 ; \mathrm{F}\right.$ participants with high remoteness $(1,19)=2.28, p=.14)$. However, participants with low remoteness scores showed higher alpha desynchronization in the temporo-parietal region than participants with high remoteness scores $(\mathrm{F}(1,19)=10.63, \mathrm{p}=.004)$. While participants with low remoteness scores showed alpha desynchronization in the temporo-parietal region from the beginning of activation period $\left(\mathrm{F}_{\text {Linear }}(1,19)<1\right)$, a linear decrease in the alpha synchronization was observed in participants with high remoteness scores $\left(\mathrm{F}_{\text {Linear }}(1,19)=4.33, \mathrm{p}=.05\right)$.

To better characterize the Condition x ROI x Time Window x Remoteness interaction, we conducted ANOVAs on the TRP for each time windows separately considering Condition $\left(\mathrm{C}_{\mathrm{C}}\right.$ vs. $\left.\mathrm{C}_{\mathrm{FP}}\right)$ and $\mathrm{ROI}$ (frontal vs. temporo-parietal) as within-subjects factors and Remoteness (participants with high remoteness vs. participants with low remoteness scores) as a betweensubjects factor. Results showed that the Condition x ROI x Remoteness interactions were not significant for the four first time windows (all ps > .30). Nevertheless, we found significant Condition $x$ ROI $x$ Remoteness interactions for the fifth $\left(F(1,19)=7.86, p=.01, \eta_{p}{ }^{2}=0.29\right)$, the sixth $\left(\mathrm{F}(1,19)=12.24, \mathrm{p}=.002, \eta_{\mathrm{p}}{ }^{2}=0.39\right)$ and the seventh $\left(\mathrm{F}(1,19)=5.51, \mathrm{p}=.03, \eta_{\mathrm{p}}{ }^{2}\right.$ $=0.22)$ time windows. Given that effect size was higher for the sixth time window (-750 to $550 \mathrm{~ms}$ ), we conducted further post hoc comparisons restricted to this time window. Post hoc comparisons in the frontal ROI confirmed that participants with high remoteness scores showed higher alpha synchronization than participants with low remoteness scores in the $\mathrm{C}_{\mathrm{C}}$ $(\mathrm{F}(1,19)=4.98, \mathrm{p}=.04)$ but not in the $\mathrm{C}_{\mathrm{FP}}(\mathrm{F}(1,19)<1)$. In addition, participants with high remoteness scores showed higher alpha desynchronization in the temporo-parietal ROI than 
participants with low remoteness scores in the $\mathrm{C}_{\mathrm{FP}}(\mathrm{F}(1,19)=12.69, \mathrm{p}=.002)$ but not in the $\mathrm{C}_{\mathrm{C}}(\mathrm{F}(1,19)=2.00, \mathrm{p}=.17)$.

\section{Discussion}

The present study aimed to investigate the neural bases of the processes involved in overcoming fixation effects during creative idea generation. Using the AU task adapted for EEG recording, we examined whether participants' ability to provide original ideas was related to alpha power changes in both frontal and temporo-parietal regions. Critically, for half of the objects, the classical use of the object was primed orally, and a picture of the classical use was presented visually to increase functional fixedness (Fixation Priming condition). For the other half, only the name of the object and a picture of the object were provided to the participants (control condition).

As expected, priming the classical use of an object before the generation of creative alternative uses of this object impeded participants' performances in terms of remoteness. Our results are consistent with those obtained in the field of problem solving (Adamson, 1952; Duncker, 1945; German \& Barrett, 2005; German \& Deyfeter, 2003) and demonstrate that functional fixedness also occurs during the generation of multiple creative ideas. Note that functional fixedness seems to be short-lived in this context given that only the first answer provided by the participants was less remote in the Fixation Priming condition than in the Control condition. These results might also suggest that participants use compensatory strategies to overcome fixation effects during the course of creative idea generation.

Critically, we observed a strong negative correlation between the remoteness scores in the AU task and the interference scores in the Hayling Sentence Completion Test. In line with findings from both behavioral and neuroimaging studies (Beaty et al., 2015; Camarda et al., in press; Cassotti et al., 2016), this correlation suggests that the inhibition of automatic close 
semantic associations might be a core process of creative ideation and critical to the generation of distant semantic associations. Indeed, the Hayling Sentence Completion Test is one of the most commonly used tests to measure inhibitory control ability in neuropsychological assessments (Pérez-Pérez, 2016). Importantly, this test has been previously associated with an increase in brain activation in regions known to be involved in inhibitory control, such as the inferior frontal gyrus (Collette et al., 2001; Robinson et al., 2015) and the orbitofrontal cortex (Collette et al., 2001; Volle et al., 2012). However, it should be noted that this task might involve other processes critical for creative ideation. In particular, the Hayling Sentence Completion Test also requires the generation of remote (but unrelated) semantic associations. Thus, further EEG studies are needed to disentangle the respective contributions of inhibitory control and semantic associations during the generation of creative ideas using other measurements of inhibitory control, such as the Stroop task (Benedeck et al., 2012).

Electrophysiological data provide additional support for the involvement of cognitive control in creative idea generation. Consistent with previous studies, we found that taskrelated alpha power was higher for participants with high remoteness scores than for participants with low remoteness scores (Benedek et al., 2011; Benedek et al., 2014; Fink et al., 2006). A fine-grained analysis of frontal and temporo-parietal alpha power revealed different patterns of activity depending on the remoteness score of the participants. While frontal alpha synchronization was maintained across all successive time windows for participants with high remoteness scores, the frontal alpha synchronization decreased in participants with low remoteness scores. Given that alpha synchronization reflects cortical arousal and, in particular, top-down processes that regulate and inhibit bottom-up processes or irrelevant semantic associations (Benedek et al., 2011; 2014; Klimesh et al., 2007), our results further suggest that highly expansive participants might use top-down control and executive 
processes to generate creative ideas, which is in line with findings from previous studies (Benedek et al., 2011; 2014; Cassotti et al., 2016; Kleibeuker et al., 2016).

As opposed to what we observed in the control condition, in the Fixation Priming condition, in which functional fixedness was maximal, both participants with high and low remoteness scores maintained frontal alpha synchronization throughout the period preceding their answer. This result suggests that participants might rely on top-down control to overcome fixation when functional fixedness increases regardless of their remoteness level (Benedek et al., 2011; Benedek et al., 2014; Beaty, et al., 2014; 2016). Thus, differences in remoteness levels in this specific context are not rooted in differences in top-down processes recruitment and maintenance per se. We did, however, observe that participants with high and low remoteness scores displayed different patterns of alpha power within the temporo-parietal regions in the Fixation Priming condition. Whereas participants with high remoteness scores maintained alpha synchronization in the temporo-parietal regions throughout the creative idea generation period, participants with low remoteness scores displayed alpha desynchronization in the same regions during this period. In light of the fact that alpha synchronization and desynchronization in the temporo-parietal regions reflect, respectively, high-level internal processes leading to the combination of distant semantic elements and bottom-up processes leading to semantic associations based on environmental cues (Benedek et al., 2011; Fink et al., 2006; Fink, et al., 2009; Von Stein \& Sarnthein, 2000), we suspect that participants with high remoteness scores might generate more creative ideas than participants with low remoteness scores because they rely more on internal semantic associations and selection processes.

Some limitations of this study should be acknowledged. First, even though we used a paradigm adapted for EEG recording (Fink et al., 2006), participants were only asked to complete the Alternative Uses Task. However, previous studies have revealed that task- 
related alpha power can be modulated by the nature of the creative task proposed to the participants (e.g., Benedek et al., 2012; 2014). Thus, further studies are required to determine whether the results observed in the present study can be generalized to other contexts inducing fixation effects. (Adamson, 1952; Duncker, 1945; Agogué, Kazakçi, et al., 2014; Agogué, Poirel, et al., 2014). In addition, alpha synchronization in the frontal regions might reflect not only cognitive control over prepotent responses (here, inhibitory control of the classical use of an object) but also other executive functions such as working memory or flexibility. Note, however, that one study demonstrated that depleting cognitive control but not working memory affected the ability to generate creative ideas (Camarda et al., in press). In light of this finding and findings from numerous studies showing that cognitive control is the core mechanism allowing one to overcome bias in reasoning (Houdé et Borst, 2014; 2015), we are confident that frontal alpha synchronization in our study reflected the recruitment of cognitive control.

Several previous EEG studies of creative ideation have reported that the upper alpha band is more sensitive to high task demands than the lower alpha band (Fink \& Benedek, 2014; Klimesch, Sauseng, \& Hanslmayr, 2007). Nevertheless, a difference between the upper and lower alpha bands was not observed in the present study. Although this result is in line with a recent EEG investigation reporting similar patterns of alpha synchronization between the lower and upper alpha bands when the need for internal processing (high vs low) was systematically manipulated (Benedek et al., (2011), further research is required to clarify the reason for these discrepancies.

To conclude, our study is the first to investigate the neural bases of the processes involved in overcoming fixation effects during the generation of creative ideas. Our findings provide additional evidence for the role of cognitive control in creative ideation while suggesting that cognitive control is not enough to generate new ideas. Indeed, in a context in 
which functional fixedness is maximal (by priming the typical use of an object), the ability to generate creative ideas is related to cognitive control and the strategies used to create new semantic associations - i.e., participants who were better at creating distant semantic associations were those who used strategies relying on high-level internal processes to combine distant elements. Although the present study did not aim at investigating the relationship between fluid intelligence, creative ideation and inhibitory control, it would be interesting, in regard to the emergent literature on this topic (Beaty et al., 2014; Benedek, et al., 2012; Vartanian, 2009), to examine how these factors each contribute to creative performance, the ability to overcome fixation effects and alpha-power-related changes in both frontal and temporo-parietal regions. 


\section{References}

Adamson, R. E. (1952). Functional fixdness as related to problem solving: a repetition of three experiments. Journal of Experimental Psychology, 44(4), 288-291.

Amabile, T. M., \& Pillemer, J. (2012). Perspectives on the Social Psychology of Creativity. The Journal of Creative Behavior, 46(1), 3-15.

Agogué, M., Le Masson, P., Dalmasso, C., Houdé, O., \& Cassotti, M. (2015). Resisting classical solutions: The creative mind of industrial designers and engineers. Psychology of Aesthetics, Creativity, and the Arts, 9(3), 313.

Agogué, M., Poirel, N., Pineau, A., Houdé, O., \& Cassotti, M. (2014). The impact of age and training on creativity: a design-theory approach to study fixation effects. Thinking Skills and Creativity, 11, 33-41.

Agogué, M., Kazakçi, A., Hatchuel, A., Le Masson, P., Weil, B., Poirel, N., \& Cassotti, M. (2014). The impact of type of examples on originality: Explaining fixation and stimulation effects. The Journal of Creative Behavior, 48, 1-12.

Amabile, T. (1982). Social psychology of creativity: A consensual assessment technique. (1982). Journal of Personality and Social Psychology, 43, 997-1013.

Benedek, M., Bergner, S., Könen, T., Fink, A., \& Neubauer, A. C. (2011). EEG alpha synchronization is related to top-down processing in convergent and divergent thinking. Neuropsychologia, 49(12), 3505-3511.

Benedek, M., Franz, F., Heene, M., \& Neubauer, A. C. (2012). Differential effects of cognitive inhibition and intelligence on creativity. Personality and Individual Differences, 53, 480-485.

Benedek, M., Schickel, R. J., Jauk, E., Fink, A., \& Neubauer, A. C. (2014). Alpha power increases in right parietal cortex reflects focused internal attention. Neuropsychologia, $56,393-400$.

Beaty, R. E., Benedek, M., Barry Kaufman, S., \& Silvia, P. J. (2015). Default and Executive Network Coupling Supports Creative Idea Production. Scientific Reports, 5, 10964.

Beaty, R. E., Benedek, M., Silvia, P. J., \& Schacter, D. L. (2016). Creative Cognition and Brain Network Dynamics. Trends in Cognitive Sciences, 20(2), 87-95. 
Beaty, R. E., Silvia, P. J. Nusbaum, E. C., Jauk, E., \& Benedek, M. (2014). The role of associative and executive processus in creative cognition. Memory and cognition, 47(7), 1186-1197.

Burgess, and Shallice. (1996). Response suppression, initiation and strategy use fallowing frontal lobe lesions. Neuropsychologia, 34(4), 263-273.

Camarda, A., Borst, G., Agogué, M., Habib, M., Weil, B., Houdé, O., \& Cassotti, M (2017). Do we need inhibitory control to be creative ? Evidence from a dual - task paradigme. Psychology of Aesthetics, Creativity and the Art. (In press).

Cassotti, M., Agogué, M., Camarda, A., Houdé, O., \& Borst, G. (2016). Inhibitory control as a core process of creative problem solving and idea generation from childhood to adulthood. New directions for child and adolescent development, 151, 61-72.

Collette, F., Van der Linden, M., Delfiore, G., Degueldre, C., Luxen, A.,\&Salmon, E. (2001). The functional anatomy of inhibition processes investigated with the Hayling task. NeuroImage, 14, 258-267.

Cooper, N. R., Croft, R. J., Dominey, S. J. J., Burgess, A. P., \& Gruzelier, J. H. (2003). Paradox lost? Exploring the role of alpha oscillations during externally vs. Internally directed attention and the implications for idling and inhibition hypotheses. International Journal of Psychophysiology, 47, 65-74.

Delorme, A., \& Makeig, S. (2004a). EEGLAB: an open source toolbox for analysis of singletrial EEG dynamics including independent component analysis. Journal of neuroscience methods, 134, 9-21.

Delorme, A., \& Makeig, S., (2004b). EEGLAB: an open source toolbox for analysis of singletrial EEG dynamics including independent component analysis. (2004). Journal of neuroscience methods, 134(1), 9-21.

De Neys, W., Vartanian, O., \& Goel, V. (2008). Smarter than we think: When our brains detect that we are biased. Psychological Science, 19, 483-489.

Duncker, K., \& Lees, L. S. (1945). On problem-solving. Psychological Monographs, 58(5).

Fink, A., \& Benedek, M., (2014). EEG alpha power and creative ideation. Neuroscience and Biobehavoral Reviews, 44, 111-123. 
Fink, A., Benedek, M., Grabner, R. H., Staudt, B., \& Neubauer, A. C. (2007). Creativity meets neuroscience: Experimental tasks for the neuroscientific study of creative thinking. Methods, 42(1), 68-76.

Fink, A., Grabner, R. H., Benedek, M., \& Neubauer, A. C. (2006). Divergent thinking training is related to frontal electroencephalogram alpha synchronization. European Journal of Neuroscience, 23(8), 2241-2246.

Fink, A., Grabner, R. H., Benedek, M., Reishofer, G., Hauswirth, V., Fally, M., Neuper, C., Ebner, F., \& Neubauer, A. C. (2009). The creative brain: Investigation of brain activity during creative problem solving by means of EEG and FMRI. Human Brain Mapping, 30(3), 734-748.

Fink, A., Grabner, R. H., Gebauer, D., Reishofer, G., Koschutnig, K., \& Ebner, F. (2010). Enhancing creativity by means of cognitive stimulation: Evidence from an fMRI study. NeuroImage, 52(4), 1687-1695.

German, T.P., \& Barrett, H.C. (2005). Functional fixedness in a technologically sparse culture. Psychological Science, 16, 1-5.

German, T. P., \& Defeyter, M. A. (2000). Immunity to functional fixedness in young children. Psychonomic Bulletin \& Review, 7(4), 707-712.

Grabner, R. H., Fink, A., \& Neubauer, A. C. (2007). Brain correlates of self-rated originality of ideas: Evidence from event-related power and phase-locking changes in the EEG. Behavioral Neuroscience, 121(1), 224-230.

Guilford, J. P. (1967). The nature of human intelligence. New York : McGraw-Hill.

Halliday, D.M., Rosenberg, J.R., Amjad, A.M., Breeze, P., Conway, B.A., Farmer, S.F., 1995.A framework for the analysis of mixed time series/point process data-theory and application to the study of physiological tremor, single motor unit discharges and electromyograms. Progress in Biophysics and Molecular Biology, 64, 237-278.

Hao, N., Wu, M., Runco, M. A., \& Pina, J. (2015). More mind wandering, fewer original ideas: Be not distracted during creative idea generation. Acta psychologica, 161, 110116.

Houdé, O., \& Borst, G. (2014). Measuring inhibitory control in children and adults: Brain imaging and mental chronometry. Frontiers in Developmental Psychology, 5:616. 
Houdé, O., \& Borst, G. (2015). Evidence for an inhibitory-control theory of the reasoning brain. Frontiers in Human Neuroscience, 9 :148.

Jansson, D.G., \& Smith, S.M. (1991). Design fixation, Design Studies, 12 (1), 3-11.

Jauk, E., Benedek, M., \& Neubauer, A. C. (2012). Tackling creativity at its roots: Evidence for different patterns of EEG alpha activity related to convergent and divergent modes of task processing. International Journal of Psychophysiology, 84(2), 219-225.

Karmiloff-Smith, A. (1990). Constraints on representational change: Evidence from children's drawing. Cognition, 34(1), 57-83.

Kleibeuker, S. W., De Dreu, C. K., \& Crone, E. A. (2016). Creativity development in adolescence: Insight from behavior, brain, and training studies. New directions for child and adolescent development, 151, 73-84.

Klimesch, W., Sauseng, P., \& Hanslmayr, S. (2007). EEG alpha oscillations: the inhibitiontiming hypothesis. Brain research reviews, 53(1), 63-88.

Kohn, N. W., \& Smith, S. M. (2011). Collaborative xation: Effects of others' ideas on brainstorming. Applied Cognitive Psychology, 25(3), 359-371.

Martindale, C. (1999). Biological bases of creativity. Handbook of creativity, 2, 137-152.

Martindale, C., \& Hasenfus, N. (1978). EEG differences as a function of creativity, stage of the creative process, and effort to be original. Biological Psychology, 6(3), 157-167.

Mullen, T. R., Kothe, C. A. E., Chi, M., Ojeda, A., Kerth, T., Makeig, S., Jung, T. J., Cauwenberghs, G. (2015). Real-time neuroimaging and cognitive monitoring using wearable dry EEG. IEEE Transactions on Biomedical Engineering, 62(11), 25532567.

Niedermeyer, E., \& da Silva, F. L. (Eds.). (2005). Electroencephalography: basic principles, clinical applications, and related fields. Lippincott Williams \& Wilkins.

Pérez-Pérez, A., Matias-Guiu, J. A., Cáceres-Guillén, I., Rognoni, T., Valles-Salgado, M., Fernández-Matarrubia, M., Morenon-Ramos, T., and Matías-Guiu, J. (2016). The Hayling Test: Development and Normalization of the Spanish Version. Archives of Clinical Neuropsychology, 31(5), 411-419.

Pfurtscheller, G., \& Da Silva, F. L. (1999). Event-related EEG/MEG synchronization and desynchronization: basic principles. Clinical neurophysiology, 110(11), 1842-1857. 
Pfurtscheller, G., Stancak, A., \& Neuper, C. (1996). Event-related synchronization (ERS) in the alpha band - an electrophysiological correlate of cortical idling: a review. International journal of psychophysiology, 24(1), 39-46.

Purcell, T., \& Gero, J. S. (1996). Design and other types of fixation. Design Studies, 17(4), 363-383.

Radel, R., Davranche, K., Fournier, M., \& Dietrich, A. (2015). The role of (dis) inhibition in creativity: Decreased inhibition improves idea generation. Cognition, 134, 110-120.

Sauseng, P., Klimesch, W., Schabus, M., \& Doppelmayr, M. (2005). Fronto-parietal coherence in theta and upper alpha reflect central executive functions of working memory. International Journal of Psychophysiology, 57, 97-103.

Smith, S.M., Ward, T.B., \& Schumacher, J.S. (1993). Constraining effects of examples in a creative generation task. Memory and Cognition, 21, pp. 837-845.

Sternberg, R. J., \& Lubart, T. I. (1991). An investment theory of creativity and its development. Human Development, 34(1), 1-31.

Schwab, D., Benedek, M., Papousek, I., Weiss, E. M., \& Fink, A. (2014). The time-course of EEG alpha power changes in creative ideation. Frontiers in Human Neuroscience, 8.

Storm, B. C. \& Angello, G. (2010). Overcoming fixation: Creative problem solving and retrieval-induced forgetting. Psychological Science, 21, 1263-1265.

Vartanian, O. (2009). Variable attention facilitates creative problem solving. Psychology of Aesthetics, Creativity, and the Arts, 3, 57-59.

Volle, E., De Lacy Costello, A., Coates, L. M., McGuire, C., Towgood, K., Gilbert, S., Kinkingnehun, S., McNeil, J. E., Greenwood, R., Papps, B., Can Den Broeck, M., and Burgess, P. W. (2012). Dissociation between Verbal Response Initiation and Suppression after Prefrontal Lesions. Cerebral Cortex, 22(10), 2428-2440.

Von Stein, A., \& Sarnthein, J. (2000). Different frequencies for different scales of cortical integration: from local gamma to long range alpha/theta synchronization. International journal of psychophysiology, 38(3), 301-313.

Ward, T. B. (1994). Structured imagination: The role of category structure in exemplar generation. Cognitive psychology, 27(1), 1-40. 
Zabelina, D. L., \& Robinson, M. D. (2010). Creativity as flexible cognitive control. Psychology of Aesthetics, Creativity, and the Arts, 4(3), 136-143. 
Figure captions

Figure 1. Schematic of the time course of the experiment.

Figure 2. Schematic representation of the electrode positions used for the 1) left frontal ROI, 2) right frontal ROI, 3) left temporo-parietal ROI and 4) right temporo-parietal ROI.

Figure 3. (A) Impact of functional fixedness on the remoteness of the first and last answers given in Control and Fixation Priming conditions. (B) Individual mean interference RTs of the Hayling Sentence Completion Test as a function of the individual mean remoteness scores in the AU task. The best-fitting line is depicted in black.

Figure 4. Alpha task related power (TRP) observed according experimental conditions and participants' remoteness scores, additionally illustrated with TRP topographical maps (top view) on which the number on the abscissa axes correspond to the different time windows analyzed ([1: $-1250 \mathrm{~ms}$ to $-1050 \mathrm{~ms}] ;$ [2: $-1150 \mathrm{~ms}$ to $-950 \mathrm{~ms}]$; [3: $-1050 \mathrm{~ms}$ to $-850 \mathrm{~ms}]$; [4: $-950 \mathrm{~ms}$ to $-750 \mathrm{~ms}] ;[$ [5: $-850 \mathrm{~ms}$ to $-650 \mathrm{~ms}] ;[6:-750 \mathrm{~ms}$ to $-550 \mathrm{~ms}]$; [7: $-650 \mathrm{~ms}$ to -450 $\mathrm{ms}]$; [8: $-550 \mathrm{~ms}$ to $-350 \mathrm{~ms}]$; [9: $-450 \mathrm{~ms}$ to $-250 \mathrm{~ms}])$. 